\title{
OGM Diffusion des OGM et impact pour les agriculteurs : quels sont les liens ?
}

\section{GM foods The spread of GM foods and how it affects farmers: what are the connections?}

Oléagineux, Corps Gras, Lipides. Volume 8, Numéro 3, 204-10, Mai - Juin 2001, La filière

Auteur(s) : Stéphane LEMARIE, Inra, Département d'économie et sociologie rurales, Equipe SERD, Laboratoire de Grenoble, Université Pierre-Mendès-France, BP 47, 38040 Grenoble Cedex 9.

Résumé : Les OGM actuellement sur le marché présentent des innovations sur des fonctions de protection des plantes. Selon les problèmes de protection des plantes, les gains des agriculteurs liés à l'adoption des OGM sont très variables. Cette hétérogénéité pose d'importants problèmes méthodologiques pour pouvoir estimer correctement la distribution des gains pour l'ensemble des agriculteurs. Une synthèse de plusieurs études américaines est réalisée ici et met bien en évidence la diversité des méthodes utilisées, chacune d'elles présentant ses avantages et ses limites. Globalement, si la diffusion des OGM est un fait indéniable, les études ne permettent pas de tirer des conclusions aussi nettes quant à l'impact des OGM pour tous les agriculteurs qui adoptent ces produits, et cela en particulier pour le soja. Cette absence de conclusion s'explique en grande partie par la difficulté à mesurer certains facteurs qui influencent le comportement d'adoption, comme par exemple la simplification de la conduite des cultures. En contrepartie, il faut noter que les études présentées ici ne prennent pas en compte un certain nombre d'effets indirects qui peuvent faire diminuer les gains des agriculteurs.

Mots-clés : innovation, adoption, diffusion, OGM, protection des plantes.

Summary : The GMOs currently on the market offer innovations on plant protection characters. The economic gain provided by the GMO is variable and depends first of all on the precise plant protection problem faced by the farmers. The heterogeneity raises important methodological problems for the analysis of the distribution of the economic gains for the farmers. A synthesis of several American studies is proposed here and shows the diversity of the methods used, each of them having its own advantages and drawbacks. Even though the diffusion level of GMO is an undeniable fact, the studies on the gain for the adopter do not lead to as undeniable conclusions, in particular in the case of soybean. Such a conclusion can be explained by the difficulty in measuring some factors that may influence the adoption behaviour (in particular those linked with the simplification of the farming practices). On the other hand, one have to mention that the studies presented here do not take into account a series of factors that may lead to diminishing returns for the farmers in the medium or the long run.

Keywords : innovation, adoption, diffusion, GMO, plant protection. 


\section{ARTICLE}

Les OGM ont connu une diffusion rapide aux États-Unis en l'espace de quelques années. D'une manière générale, une telle vitesse de diffusion laisse penser que l'innovation présente un intérêt économique indéniable pour les adopteurs. Pourtant, les OGM soulèvent très souvent des interrogations quant à leur impact économique, même lorsqu'on se limite au niveau de l'agriculteur. Dans cet article, nous tentons de confronter les observations faites sur la diffusion des innovations avec les résultats des études sur l'impact économique de ces innovations sur les gains des agriculteurs. Une telle façon de poser le problème nous conduit naturellement à travailler sur des cas où il y a eu une diffusion effective de ces produits. En l'occurrence, nous travaillerons sur le cas des États-Unis. Compte tenu de la littérature existante, cette synthèse couvre essentiellement l'impact des OGM sur la marge brute par culture au travers du rendement et des dépenses en semences et pesticides. Certaines études prennent également en compte l'économie liée à la réduction du nombre de passages.

Un bref rappel des caractéristiques des produits et de l'état de leur diffusion est d'abord réalisé. Un modèle graphique est ensuite présenté pour illustrer le lien entre la diffusion et l'impact de l'innovation pour les agriculteurs. Une synthèse de quelques résultats principaux sur l'impact des OGM est enfin réalisée. Une discussion plus large sur l'ensemble des effets économiques liés à la diffusion des OGM est abordée en conclusion.

\section{Caractéristiques des produits OGM et coût d'accès}

\section{Des produits qui représentent de nouveaux programmes de protection des plantes}

Jusqu'à présent, les principaux produits OGM mis sur le marché ont présenté deux caractères (éventuellement combinés) ${ }^{2}$ : une tolérance à des herbicides (désignée généralement par HT), et/ou une résistance face à certains insectes (désignée généralement par $I R$ ou $B t$ ). À chaque fois, la semence OGM présente une innovation sur un caractère lié à la protection des plantes, et son adoption se traduit par un changement de programme de protection des plantes. Autrement dit, l'agriculteur réalise plus que le simple remplacement d'une semence conventionnelle par une semence OGM. Comme nous le verrons plus loin, le choix d'une semence OGM est réalisé en comparant la profitabilité des deux types de programme de protection des plantes, le coût de la semence n'étant qu'une partie.

Nous ne pouvons pas ici entrer dans le détail des différents types de remplacement compte tenu de la multiplicité des programmes conventionnels ${ }^{3}$ de protection de plantes. Prenons simplement le cas du remplacement d'un programme conventionnel intensif par un programme OGM (tableau 1). Dans le cas des semences HT, l'emploi d'herbicide sélectif post-levée dans les programmes conventionnels peut être remplacé par l'emploi d'herbicide total. Dans le cas des semences IR, l'emploi d'insecticide dans les programmes conventionnels est nettement réduit car la semence apporte le caractère de résistance à l'insecte.

\section{Coût d'accès aux semences OGM}

Rappelons qu'à la différence des variétés de semences conventionnelles, les variétés OGM sont proposées par deux innovateurs : l'entreprise de biotechnologie, qui fournit le caractère lié à la transformation (résistance à un insecte, tolérance à un herbicide), et le semencier, qui fournit la 
variété dans laquelle il insère l'événement de transformation. L'accès des semenciers aux événements de transformation nécessite la signature d'un accord de licence avec l'entreprise de biotechnologie dans lequel sera fixé le niveau des royalties reversé sur chaque sac de semence OGM vendu. Bien qu'on ne connaisse pas le détail de ces accords, il est généralement admis que ces royalties représentent l'essentiel du supplément de prix payé sur la semence par l'agriculteur.

Les suppléments de prix payés sur les semences OGM sont variables selon les événements (tableau 2). Ils sont déterminés essentiellement par deux facteurs :

- le gain réalisé par rapport aux programmes conventionnels concurrents. Celui-ci est très élevé dans le cas du coton IR où plusieurs traitements insecticides peuvent être économisés. En revanche, il est moins élevé dans le cas du maïs IR où un seul traitement est suffisant en conventionnel pour lutter contre les attaques de pyrale (si celles-ci sont assez fortes);

- dans le cas des événements HT, le coût du programme OGM dépend du supplément de prix payé sur la semence et du prix de l'herbicide total. Dans le cas des semences $L L$, Aventis utilise la semence comme un moyen pour mettre en valeur son herbicide, et c'est donc sur ce dernier que la marge est réalisée. Dans le cas des semences RR, le brevet sur le Roundup étant tombé dans le domaine public, Monsanto complète la faible marge réalisée sur la vente de l'herbicide par une marge sur la semence OGM.

Deux autres facteurs auraient pu influencer le prix des semences OGM, mais ne présentent pas d'effets significatifs dans le cas présent :

- les coûts de production. Ceux-ci sont très similaires entre la production de semences OGM et la production de semences conventionnelles, ils ne permettent pas d'expliquer les différences de prix de vente de la semence ;

- la possibilité de discriminer les prix selon les régions. Compte tenu de l'hétérogénéité des problèmes de protection des plantes, l'intérêt à adopter des OGM peut être nettement différent d'une région à l'autre. Dans ces conditions, l'innovateur pourrait avoir intérêt à discriminer les prix en appliquant une tarification plus élevée dans les régions où les problèmes de protection des plantes sont plus forts. Une telle pratique n'a cependant pas été observée.

\section{Diffusion des OGM}

La diffusion des OGM a été très rapide entre les années 1996 et 1999 (tableau 3), I'année 2000 marquant plutôt un repli par rapport à l'année 1999. Quelle que soit l'interprétation donnée à cette dernière évolution, les produits mis sur le marché semblent avoir atteint un plafond de diffusion. Les écarts entre les niveaux des plafonds atteints laissent penser que les événements proposés sur le marché ne présentent pas globalement le même intérêt. En particulier, tout laisse penser que la tolérance au Roundup présente plus d'intérêt sur le soja que sur le maïs. Un modèle graphique simple est proposé plus loin pour expliquer le déterminisme du plafond de diffusion à partir des gains réalisés par les agriculteurs. 


\section{Modèle graphique simple pour représenter l'adoption par les agriculteurs}

On se limite ici à l'analyse du plafond de diffusion ${ }^{4}$. Lorsque le niveau de diffusion est proche du plafond, on peut penser que tous les agriculteurs pour lesquels l'adoption présente un intérêt ont adopté. Implicitement, nous supposons ici que le déroulement de la diffusion pendant les toutes premières années a peu d'influence sur le niveau du plafond qui est atteint. Autrement dit, on peut étudier le niveau du plafond qui est atteint indépendamment des événements qui se sont produits auparavant.

Comme nous l'avons indiqué plus haut, les produits présentent des innovations sur des fonctions de protection des plantes, et le choix de l'agriculteur ne concerne pas seulement la semence OGM mais tout un programme de protection des plantes. Par conséquent, on peut penser que l'agriculteur raisonne en comparant la rentabilité de différents programmes de protection des plantes.

La figure 1 donne une représentation graphique de la comparaison faite entre deux programmes de protection des plantes (A et B). La partie supérieure du graphique donne une indication du profit par hectare dégagé à partir de chaque programme de protection des plantes en fonction de l'ampleur des problèmes rencontrés ${ }^{5}$. Les courbes sont nécessairement décroissantes pour l'une ou l'autre des deux raisons suivantes:

- quand les problèmes deviennent plus aigus, le programme retenu n'est plus aussi efficace et on observe alors une baisse de rendement ;

- le programme retenu peut garder toute son efficacité technique (maintien du rendement), mais il est nécessaire d'augmenter les doses et par conséquent les dépenses en input.

Plus une courbe décroît rapidement et moins le programme est efficace du point de vue technique. Pour le dire autrement, les programmes correspondant à des courbes fortement décroissantes peuvent être qualifiés de programmes bas de gamme alors que les programmes correspondant à des courbes faiblement décroissantes peuvent être qualifiés de programmes haut de gamme.

Si l'on suppose que l'agriculteur choisit le programme qui lui offre le meilleur profit, alors on peut définir un niveau limite sur le problème de protection des plantes au-dessus duquel on préférera le programme haut de gamme et en dessous duquel on préférera le programme bas de gamme. Lorsque la loi de distribution sur les problèmes de protection des plantes est connue (partie basse de la figure 1), il est possible de donner une estimation du pourcentage des agriculteurs choisissant le programme haut de gamme (partie verte ${ }^{6}$ sur la figure 1 ).

On peut vérifier que les courbes de demande pour chacun des deux programmes sont décroissantes avec le coût de ce programme. Lorsque le prix des inputs utilisés pour un programme augmente, toute la courbe de profit se déplace vers le bas. Le niveau limite de basculement va alors se déplacer à l'avantage de la technologie concurrente. Par exemple, si le prix du programme A augmente, le niveau limite va se déplacer vers la droite et ce programme sera donc adopté par moins d'agriculteurs.

En absence de problèmes de protection des plantes, les différents programmes conduisent au même rendement. L'écart de profit entre les deux programmes (delta dans la figure 1) reflète donc l'écart de coût entre les deux types de programme. Lorsque le coût des deux programmes est le même, 
l'écart delta est nul et tous les agriculteurs ont intérêt à adopter le programme haut de gamme A. Autrement dit, indépendamment du coût du programme, tous les agriculteurs ne tirent pas le même bénéfice d'un programme de protection des plantes. L'agriculteur le plus exposé aura toujours tendance à mieux valoriser le programme haut de gamme que le programme bas de gamme.

Si certains de ces programmes sont fondés sur des produits commercialisés par des entreprises, celles-ci auront tendance à fixer un prix qui leur permettra de dégager le meilleur profit possible en tenant compte des éventuelles réactions des concurrents proposant le même programme ou d'autres programmes concurrents. II n'est pas possible ici de développer cette partie dans le détail, mais on peut observer que, dans un grand nombre de cas, le prix du programme haut de gamme n'a pas forcément intérêt à être fixé de façon à ce qu'il prenne tout le marché aux dépens des autres programmes ${ }^{7}$.

\section{Synthèse de quelques résultats sur l'impact direct des OGM pour les agriculteurs}

Le modèle présenté plus haut nous permet de comprendre comment des programmes bas de gamme et haut de gamme peuvent co-exister sur le marché du fait de l'hétérogénéité des gains pour les agriculteurs. Dans ces conditions, il est tout à fait possible que la diffusion des OGM reste partielle.

Nous allons tenter de mettre à l'épreuve ce modèle. Plus précisément, l'objectif est de voir s'il y a une cohérence entre les niveaux de diffusion observés au cours des dernières années (1999 et 2000) et la distribution des gains pour les différents agriculteurs. Par exemple, est-ce que les études ont tendance à montrer que l'adoption de soja HT est avantageuse sur $50 \%$ des surfaces américaines de soja ? Une telle question nous oblige à retenir les études couvrant un éventail assez large et si possible représentatif des conditions de culture. Pour le dire autrement, une étude donnant par exemple des estimations de gain lié à l'adoption de soja HT uniquement dans tel comté de l'Illinois ne présente pas d'intérêt ici car il ne s'agit que d'un point dans la distribution ${ }^{8}$. Par ailleurs, comme nous l'avons souligné dans l'introduction, toutes les variables n'ont pas fait l'objet de la même attention, et les études présentées ici porte essentiellement sur le rendement et les dépenses en intrants (semences et pesticides).

\section{Résultats fondés sur des enquêtes auprès d'agriculteurs}

\section{* Enquête ARMS sur les performances des agriculteurs}

À ce jour, la principale enquête réalisée auprès des agriculteurs ayant adopté des OGM est celle réalisée par le service ARMS de I'USDA [4]. L'échantillon couvre un large éventail d'agriculteurs adopteurs et non adopteurs. Le questionnaire porte sur l'usage d'intrants, le rendement obtenu et la marge brute.

L'impact de l'utilisation des OGM ne peut être mesuré directement en comparant les marges brutes moyennes entre les exploitations ayant adopté et celles n'ayant pas adopté d'OGM. En effet, cette méthode conduit à des biais d'estimation importants si on a des raisons de penser que l'échantillon des exploitations ayant adopté des variétés OGM est différent de celui des exploitations n'ayant pas adopté. Deux arguments peuvent être avancés pour justifier cela. Premièrement, les exploitations ayant adopté sont sans doute celles qui ont le plus de problèmes d'adventices ou d'insectes, et qui ont normalement des rendements plus faibles ou des dépenses en pesticides plus fortes que la moyenne. Deuxièmement, les travaux sur la diffusion des innovations montrent que les premiers 
adopteurs sont généralement des agriculteurs très informés sur les nouveautés techniques avec des performances plus élevées. Dans ces conditions, il n'est pas possible de savoir si la différence de profit entre adopteurs et non-adopteurs est liée à l'adoption de l'innovation ou à d'autres facteurs. Les estimations présentées dans le tableau 4 ont été réalisées à partir d'un modèle économétrique où les biais statistiques liés à cet effet de sélection ont été corrigés.

Plusieurs commentaires peuvent être faits sur les résultats (tableau 4).

* Premièrement, l'adoption a les effets attendus sur la consommation de pesticides : I'adoption de coton IR conduit à une diminution de I'utilisation d'insecticide, I'adoption de soja HT conduit à une diminution de l'utilisation d'herbicide sélectif et à une augmentation de l'utilisation de glyphosate. Les effets ne ressortent en revanche pas de façon aussi nette dans le cas du coton HT.

* Deuxièmement, l'adoption conduit à un accroissement du rendement. Cette augmentation n'est pas très forte dans le cas du soja, mais il faut noter que l'année d'enquête correspond au début de la diffusion du soja HT. Or, on sait que les toutes premières variétés de soja HT n'étaient pas forcément parfaitement isogéniques avec les variétés conventionnelles, et avaient donc tendance à être moins performantes que ces dernières sur les caractères autres que le caractère $\mathrm{HT}^{9}$.

* Troisièmement, l'adoption conduit à une augmentation significative des marges réalisées sur le coton, mais ne conduit pas à des changements significatifs sur le soja. Cette dernière observation est surprenante car elle concerne la culture qui a connu la diffusion la plus forte. Le fait qu'il s'agit des toutes premières variétés de soja, comme nous l'avons observé plus haut, explique en partie ce résultat. Les auteurs expliquent également que cette estimation cache une hétérogénéité entre régions. En effet, lorsque les estimations sont faites au niveau régional, les auteurs mettent en évidence une augmentation significative du profit dans les Heartland (sud des Grands lacs), région qui représente près des trois quarts des surfaces américaines de soja. Cette explication n'est cependant pas complètement satisfaisante car la diffusion du soja dans cette région n'est pas spécialement plus forte que dans les régions voisines.

\section{* Enquête de Hubbell et al. [6] sur les propensions à payer}

Hubbell et al. [6] tentent de construire une fonction de demande en coton IR à partir des résultats d'une enquête réalisée au début de l'année 1997 auprès d'un échantillon de 210 agriculteurs. Un certain nombre de caractéristiques de l'exploitation et de l'agriculteur sont ainsi collectées. De plus, chaque non-adopteur doit indiquer si le prix de la licence est un facteur important pour expliquer son comportement et, si tel est le cas, le prix de licence en dessous duquel il serait prêt à adopter.

Les résultats (figure 2) montrent que les trois quarts des agriculteurs n'ayant pas adopté seraient prêts à le faire avec un prix de licence inférieur à 32 \$/acre. La valeur moyenne de la propension à payer de tous les agriculteurs est de $30 \$ /$ acre. Autrement dit, les agriculteurs sont dans l'ensemble perdants si on les force tous à adopter le coton IR avec une licence de $32 \$ /$ acre. Sur les États de I'Alabama et de Georgie où la diffusion du coton IR est la plus élevée (Lower South), le prix de la licence doit passer de $32 \$ /$ acre à $23 \$$ /acre pour que la diffusion passe de 50 à $75 \%$ (figure 2). Dans les autres États étudiés (Upper South, soit la Caroline du Nord et Caroline du Sud), 
le niveau de diffusion de $75 \%$ n'est atteint que lorsque la licence passe en dessous de 11 \$/acre. En d'autres termes, l'introduction d'OGM concurrents peut conduire à augmenter significativement le niveau de diffusion, si cela se traduit par une baisse significative du prix de la licence.

\section{Résultats fondés sur des modèles agronomiques}

\section{* Retour sur les limites des analyses fondées sur des données d'enquêtes}

On peut penser a priori que la méthode la plus simple et la plus robuste pour estimer l'impact des OGM consiste à demander aux agriculteurs qui les utilisent ou non ce qu'ils en pensent. Les deux exemples précédents ont montré que cette méthode pose néanmoins deux problèmes.

* Dans le cas où la performance des adopteurs est comparée à celle des non-adopteurs, on se trouve confronté au problème des biais de sélection. Ainsi, mesurer l'impact pour les adopteurs nécessiterait de connaître leur performance si on les forçait à ne pas adopter. Ceci n'est pas possible, et on se contente donc des données sur les non-adopteurs pour estimer ce que pourrait être la performance des adopteurs s'il n'adoptaient pas. Notons bien que ce problème ne se poserait pas si la population des agriculteurs n'était pas hétérogène.

* Dans le cas où l'on s'appuie sur des propensions à payer, le problème est alors de connaître le comportement qu'aurait l'agriculteur en situation réelle. Différentes raisons peuvent amener l'agriculteur à adopter ou non une variété OGM à un prix inférieur ou supérieur par rapport au prix qu'il avait indiqué en répondant à l'enquête. Ensuite, même si l'agriculteur respecte ce qu'il a annoncé, il se peut que l'impact réel soit différent de ce qu'il avait anticipé au départ, l'agriculteur ayant pu se tromper ou être trompé sur l'intérêt réel des OGM dans son exploitation.

Les analyses fondées sur des enquêtes auprès d'agriculteurs ayant montré leurs limites, une alternative possible consiste à se fonder sur des modèles agronomiques pour donner une estimation possible du rendement obtenu dans des conditions de culture données avec un itinéraire technique donné. Une fois le rendement estimé, il est possible d'estimer la marge dégagée sur la culture en décomptant le coût de l'itinéraire technique. Potentiellement, de tels modèles permettent d'estimer, pour chaque condition de culture, la marge dégagée avec différents itinéraires techniques et d'en déduire l'impact des OGM.

En se replaçant dans le cadre du raisonnement présenté plus haut avec la figure 1, le problème des analyses fondées sur les enquêtes (type ARMS) tient à ce qu'on n'a d'information que sur la courbe qui se situe le plus en haut. À l'inverse, en se fondant sur des modèles agronomiques, le travail consiste indirectement à construire intégralement les différentes courbes de profits.

Deux principales applications ont été faites en utilisant des modèles agronomiques, la première sur le cas de la résistance à la pyrale sur maïs, la seconde sur le cas du soja HT.

\section{* Cas de la résistance à la pyrale sur maïs}

Au cours de son cycle de vie, le maïs peut subir jusqu'à trois attaques de pyrale aux États-Unis. L'analyse de l'impact des variétés de maïs Bt est délicate à réaliser ex post car les attaques de pyrale sont très irrégulières selon les années. Un impact très positif sera observé suite à une forte attaque de pyrale. 
Néanmoins, si cette attaque est exceptionnelle, il se peut très bien que l'on estime que l'impact soit en moyenne négatif (le gain de rendement moyen ne compense pas le supplément de coût sur la semence).

Plusieurs chercheurs ont réalisé des études détaillées de l'intérêt économique du maïs résistant à la pyrale [7-10] à partir de modèles agronomiques. En simplifiant, on peut considérer que tous ces travaux s'appuient sur une modélisation du rendement proche de la forme suivante :

$$
y=y_{0}[1-\alpha(1-x) n]
$$

$y$ est le rendement de la culture ; $y_{0}$ est le rendement sans attaque de pyrale. Ce rendement est multiplié par un terme qui représente la chute (en pourcentage) de rendement due à l'attaque de pyrale : $n$ est le nombre moyen de pyrales par plante; a est l'impact d'un niveau d'attaque d'une pyrale par plante sur le rendement ; $x$ est le niveau d'efficacité de la technique de lutte contre la pyrale exprimé comme le pourcentage des larves détruites avant d'avoir eu un effet sur la plante.

Plusieurs gènes de résistance à la pyrale sont aujourd'hui sur le marché, avec des degrés d'efficacité variables [7] : les événements Mon810 (Monsanto) et Bt11 (Novartis) offrent une très bonne protection à tous les stades ; l'événement 176 (Ciba/Mycogen) offre une très bonne protection face à la première génération d'attaque de pyrale, mais une protection moyenne pour les deuxième et troisième générations ; enfin, l'événement DBT418 (Dekalb) offre une très bonne protection face à la première génération, et une bonne protection aux deuxième et troisième générations. Lorsqu'un produit présente une bonne efficacité (événements Mon810 et Bt11), les auteurs s'accordent pour dire que le rendement obtenu est équivalent à ce qui aurait été obtenu sans attaque de pyrale. Cela revient alors à supposer que $x=1$.

Généralement, les travaux comparent le profit obtenu avec un maïs Bt très efficace $(x=1)$ au profit obtenu avec une semence conventionnelle sans appliquer de traitement $(x=0)$. En définissant $p$ comme le prix du maïs consommation et delta le supplément de prix payé sur la semence Bt, le profit obtenu avec le maïs Bt est :

$$
\pi_{B t}=y_{0} p-\Delta
$$

et le profit obtenu avec le maïs conventionnel est :

$$
\pi_{\text {conv }}=y_{0} p(1-\alpha n)
$$

Après développement, on peut remarquer que les deux niveaux de profit sont égaux pour un niveau d'attaque :

$$
\hat{\mathrm{n}}=\mathrm{H} /\left(\alpha y_{0} p\right)
$$


En reprenant des valeurs proches de celles envisagées dans les différentes études ${ }^{10}$, on trouve que le maïs Bt devient intéressant dès lors que les attaques de pyrale dépassent 0,7 larve par plante. Cette valeur seuil est la traduction du niveau seuil de la figure 1 au-dessus duquel le programme $A$ (ici le maïs $\mathrm{Bt}$ ) devient plus intéressant que le programme $\mathrm{B}$.

Plusieurs commentaires pourraient être faits sur les développements possibles du modèle luimême ${ }^{11}$. Néanmoins, il nous semble surtout important de souligner qu'à ce jour aucune confrontation n'a été faite entre les prédictions faites par ce modèle et les données empiriques. Par exemple, aucune analyse n'a été faite pour voir si approximativement $25 \%$ de la surface en maïs américaine (soit le niveau de diffusion du maïs $\mathrm{Bt}$ ) subissait des attaques de pyrale en moyenne supérieures à 0,7 chenille/plante.

\section{* Cas du soja HT}

Dans le cas du soja, les problèmes d'adventices sont assez réguliers d'une année sur l'autre, mais variables selon les régions. L'étude réalisée par Bullock et Nitsi [11] procède en deux étapes.

D'une part, une enquête a été réalisée auprès d'un échantillon représentatif de 1400 agriculteurs du Midwest, dans le but de repérer les adventices posant le plus de problèmes sur leurs exploitations.

D'autre part, pour chaque exploitation, un logiciel simulant l'impact des programmes de traitement (Ohio Herbicide Selector Program) a été utilisé pour estimer le rendement obtenu. Une fois déduits le coût des herbicides et le coût lié à la réalisation des traitements, la comparaison des marges permet de déterminer le meilleur programme conventionnel et d'estimer le gain apporté par un programme OGM (ici : semence HT + Roundup).

Cette étude permet de dégager plusieurs résultats importants.

* Dans la grande majorité des cas, les meilleurs programmes conventionnels et le programme OGM donnent des rendements équivalents. Autrement dit, pour chaque condition de culture, le gain lié à I'adoption des OGM repose essentiellement sur une économie de dépense sur les programmes de désherbage.

* La diffusion des OGM a permis aussi aux non-adopteurs de réaliser des économies sur les dépenses de désherbage. Le coût des programmes de désherbage conventionnel a baissé en moyenne de 5 $\$$ /acre (près de $100 \mathrm{~F} / \mathrm{ha}$ ) entre 1995 et 1999 . Ceci est lié à la baisse des prix des herbicides utilisés dans les programmes conventionnels qui résulte en grande partie de la concurrence des programmes OGM. En particulier, les deux produits leaders (Imazetapyr et Chlorimuron) avant l'introduction du soja HT ont vu leur prix baisser de l'ordre de $40 \%$ en l'espace de 3 ans [3].

* Lorsque la différence de coût de désherbage entre le programme OGM et le programme conventionnel est calculée, moins de $10 \%$ des agriculteurs économisent plus de $10 \$ /$ acre, le supplément de prix à payer sur la semence. Autrement dit, ces estimations tendent à montrer que la proportion d'agriculteurs pour lesquels les OGM conduisent à des gains directs est très faible et nettement inférieure au niveau de diffusion actuel. 
Les auteurs expliquent ce résultat par le fait que les programmes OGM permettent de faciliter nettement la conduite de la culture, facteur non pris en compte dans leur analyse. En supposant par exemple que cette simplification présente une valeur de $10 \$$ /acre (près de $200 \mathrm{~F} / \mathrm{ha}$ ), l'adoption d'un programme OGM présente un intérêt pour près de $40 \%$ des agriculteurs.

Notes:

${ }^{1}$ Cet article reprend en partie les résultats d'une étude financée par le ministère de l'Agriculture et de la Pêche (référence MAP 99.A2.03.01) (encadré) [1].

${ }^{2}$ Des pommes de terre résistantes à certains virus ont été introduites en 1998. Leur diffusion a été très limitée (de l'ordre du millier d'acres) et Monsanto a récemment décidé d'en arrêter la commercialisation (Agrow $2001 ; 373$ ).

${ }^{3}$ Un programme de type conventionnel est défini, par opposition à un programme de type OGM, comme un programme dans lequel la semence utilisée est non OGM.

${ }^{4}$ Pour expliquer qu'une faible part des agriculteurs adoptent pendant les toutes premières années, plusieurs explications sont généralement apportées : la méconnaissance des caractéristiques techniques de l'innovation ; la non-technicité de certains agriculteurs et leur choix déterminé principalement par mimétisme sur le voisin ; la non-disponibilité de l'événement de transformation dans les variétés adaptées à telle ou telle zone. Le modèle proposé ici ne permet pas de prendre en compte ces trois phénomènes.

${ }^{5}$ Les courbes de la figure 1 sont linéaires pour garder une présentation simple. Le raisonnement fait ici peut aussi bien être appliqué à d'autres formes de courbe de profit en fonction des problèmes de protection des plantes, du moment qu'elles sont décroissantes.

${ }^{6}$ Le pourcentage des agriculteurs choisissant le programme haut de gamme est égal à la fréquence cumulée des agriculteurs confrontés à des problèmes de protection des plantes supérieurs au seuil limite.

${ }^{7}$ Pour augmenter les parts de marché, les entreprises doivent baisser leur prix, et il y a généralement un niveau de prix en dessous duquel les gains de part de marché ne compensent plus les pertes de marges.

${ }^{8}$ Gianessi et Carpenter ont réalisé deux synthèses [2,3] plus détaillées que celle-ci reprenant entre autres les mesures ponctuelles de l'impact des OGM.

${ }^{9}$ Cette observation a par exemple été faite par Benbrook [5] qui a observé une baisse de rendement en moyenne sur un nombre très important d'essais. L'argument d'une plus faible performance des premières variétés de soja HT est développé plus en détail par Gianessi et Carpenter [3].

${ }^{10}$ Ces valeurs sont delta $=10 \$ /$ acre, alpha $=0,05, y_{0}=140$ bushel $/$ acre et $p=2,5 \$ /$ bushel.

${ }^{11}$ Dans le cas des États-Unis, des développements sont faits dans les études mentionnées pour prendre en compte les deux à trois attaques de pyrales. Des développements intéressants pourraient être faits également pour voir l'effet de l'aversion au risque des agriculteurs. 


\section{Encadré 1}

En 1999-2000, plusieurs économistes et sociologues de I'Inra ont réalisé une étude pour le ministère de l'Agriculture et de la Pêche sur l'analyse du développement des OGM aux États-Unis (référence MAP 99.A2.03.01). Cet article reprend certains résultats du volet 1 de cette étude qui en comprend trois :

Volet 1. Le développement et l'impact des OGM agronomiques aux États-Unis : une synthèse des analyses économiques (S. Lemarié).

Volet 2. Le développement des productions agricoles présentant des caractéristiques spécifiques (J.-M. Ditner et S. Lemarié).

Volet 3. La constitution d'un " problème public » : la controverse sur les OGM et ses incidences sur la politique publique aux États-Unis (P.-B. Joly, C. Marris et O. Marcant).

L'ensemble de ce travail est disponible sur le site http://www.upmf-grenoble.fr/inra

\section{CONCLUSION}

L'objectif de cette étude a été d'établir un lien entre les observations faites sur la diffusion des OGM et une série de résultats sur l'impact des OGM pour les agriculteurs. La synthèse qui est proposée ne permet pas de répondre de manière très satisfaisante à cette question pour plusieurs raisons.

* La diffusion partielle des OGM s'explique en partie par le fait que l'impact de l'adoption pour les agriculteurs est hétérogène. De nombreuses études ont été réalisées de manière ponctuelle dans une zone limitée et ne permettent pas de faire de confrontation avec la diffusion sur l'ensemble des zones géographiques.

* Tous les types d'OGM n'ont pas fait l'objet de la même attention. En particulier, très peu de résultats sont disponibles sur le maïs tolérant à un herbicide et les seuls résultats disponibles sur le maïs résistant à la pyrale ont été établis à partir de modèle agronomique sans confrontation au terrain.

* Dans le cas du coton, un impact positif ressort très généralement en cohérence avec la diffusion observée. En revanche, dans le cas du soja tolérant à un herbicide, la diffusion importante de ce produit ne se trouve pas véritablement confirmée par les résultats des études d'impacts.

Cet article n'offre cependant pas une vue complète de l'impact des OGM pour les agriculteurs.

Premièrement, tous les facteurs pour lesquels les OGM apportent un avantage ou un inconvénient par rapport aux semences conventionnelles n'ont pas toujours été pris en compte. 
En particulier, certaines variables comme le temps passé à organiser le travail sur les cultures sont délicates à mesurer. Cet argument est généralement évoqué en creux pour expliquer l'apparente contradiction entre la diffusion rapide du soja tolérant à un herbicide et le faible impact observé pour les agriculteurs [11].

Deuxièmement, un certain nombre de facteurs peuvent avoir un effet sur les agriculteurs tout en ayant une influence faible sur la diffusion des OGM. Ceci est généralement lié à une modification de l'environnement technique ou économique.

* Modification de l'environnement technique. De tels phénomènes peuvent ne pas avoir d'effet sur le comportement d'adoption de l'agriculteur lorsqu'il s'agit d'effets à long terme. II s'agit en particulier des risques de contournement de la résistance par les insectes, des problèmes liés à la gestion de repousses résistantes à des herbicides ou, enfin, des risques de flux de gènes de résistance vers des adventices apparentés.

* Modification de l'environnement économique. Les gains de productivité permis par les OGM sur certaines exploitations peuvent conduire à une augmentation de l'offre et à une baisse des prix des produits agricoles. Cela entraîne alors un transfert de surplus de l'agriculteur vers les acteurs situés en aval et, donc, une diminution des gains pour les agriculteurs, qu'ils soient adopteurs ou non. Il est cependant difficile d'étudier ce phénomène car les prix des produits agricoles sont influencés par des facteurs autres que la diffusion des OGM (évolution des stocks mondiaux, accidents climatiques, etc.). Les économistes ont généralement recours à des modèles économiques de simulations [12-14], mais la robustesse des résultats apparaît encore assez discutable. D'une manière générale, ces travaux montrent que, après avoir pris en compte la baisse des prix des produits agricoles, les adopteurs enregistrent malgré tout une variation de surplus positive et les non-adopteurs une diminution de leurs gains.

Troisièmement, un certain nombre de facteurs indépendants peuvent avoir un effet sur la diffusion des OGM. II s'agit en particulier des évolutions de politique agricole. Frisvold et al. [15] montrent ainsi que, dans le cas du coton, la politique de soutien américaine a eu un effet favorable sur la diffusion des OGM. Un tel travail n'a pas été conduit sur le soja, mais on sait que la surface consacrée à cette culture a augmenté au cours des dernières années du fait en partie de l'évolution de la politique agricole américaine.

\section{REFERENCES}

1. LEMARIÉ S (2001). Le développement et l'impact des OGM agronomiques aux États-Unis : une synthèse des analyses économiques. In : LEMARIÉ S, DITNER JM, eds. Analyse économique du développement des cultures à base d'organismes génétiquement modifiés aux États-Unis. Grenoble, Inra. 1 : 1-50. http://www.upmf-grenoble.fr/inra

2. GIANESSI LP, CARPENTER JE (1999). Agricultural biotechnology: insect control benefits. Washington

D.C. : National Center for Food and Agricultural Policy ; 101 p. http://www.ncfap.org/pup/biotech/insectcontrolbenefits.pdf 
3. GIANESSI LP, CARPENTER JE (2000). Agricultural biotechnology: benefits transgenic soybeans. Washington D.C. : National Center for Food and Agricultural Policy ; 103 p. http://www.ncfap.org/ pup/biotech/rrsoybeanbenefits.pdf

4. FERNANDEZ-CORNEJO J, MCBRIDE WD (2000). Genetically engineered crops for pest management in US Agriculture: farm-level effects. Washington : USDA-ERS, AER-786 ; 20 p. http://www.ers.usda. gov/epubs/pdf/aer786

5. BENBROOK C (1999). Evidence of the magnitude and consequences of the Roundup Ready Soybean yield drag from university-based varietal trials in 1998. Sandpoint, Idaho : Benbrook Consulting Services, Ag BioTech InfoNet Technical Paper Number 1 ; 18 p. http://www.biotechinfo.net/RR_yield_drag_98.pdf

6. HUBBELL BJ, MARRA MC, CARLSON GA (2000). Estimating the demand for a new technology: Bt cotton and insecticide policies. Am J Agric Econ, 82 : 118-32.

7. HYDE J, MARTIN MA, PRECKEL PV, EDWARDS CR (1999). The economics of Bt corn: valuing protection from the European Corn Borer. Rev Agric Econ, 21.

8. OSTLIE KR, HUTCHISON WD, HELLMICH RL (1997). Bt Corn and European Corn Borers: long term success through resistance management. University of Minnesota, NCR602.

http://www.extension.umn.edu/distribution/cropsystems/DC7055.html

9. MARRA M, CARLSON G, HUBBELL B (1998). Economic impacts of the first crop biotechnologies. North Carolina State University. http://www.ag-econ.ncsu.edu/faculty/marra/FirstCrop/sld001.htm

10. NELSON GG, JOSLING T, BULLOCK D, ROSEGRANT M, HILL L (1999). The economics and politics of Genetically Modified Organisms in agriculture: implications for WTO 2000. Urbana- Champaign (Illinois): College of Agricultural, Consumer and Environmental Sciences, Bulletin 809; 119 p. http://web.aces.uiuc.edu/wf/GMO/GMO.pdf

11. BULLOCK DS, NITSI EI (2000). Roundup Ready Soybean technology and farm production costs: measuring the incentive to adopt. University of Illinois at Urbana-Champaign: Department of Agricultural and Consumer Economics; 36 p.

12. MOSCHINI G, LAPAN H, SOBOLEVSKY A (2000). Roundup Ready soybeans and welfare effects in the soybean complex. Agribusiness, $16: 33-55$.

13. FALCK-ZEPEDA JB, TRAXLER G, NELSON RG (2000). Rent creation and distribution from biotechnology innovations: the case of Bt cotton and herbicide-tolerant soybeans in 1997. Agribusiness, 16: 21-32.

14. FALCK-ZEPEDA JB, TRAXLER G, NELSON RG (2000). Surplus distribution from the introduction of a biotechnology innovation. Am J Agric Econ, 82 : 360-9.

15. FRISVOLD G, TRONSTAD R, MORTENSEN J (2000). Returns to Bt Cotton adoption: regional differences and commodity program effects. Western Agricultural Economics Association Annual Meeting, Vancouver, British Columbia, June 29-July 1, 2000. 
Illustrations

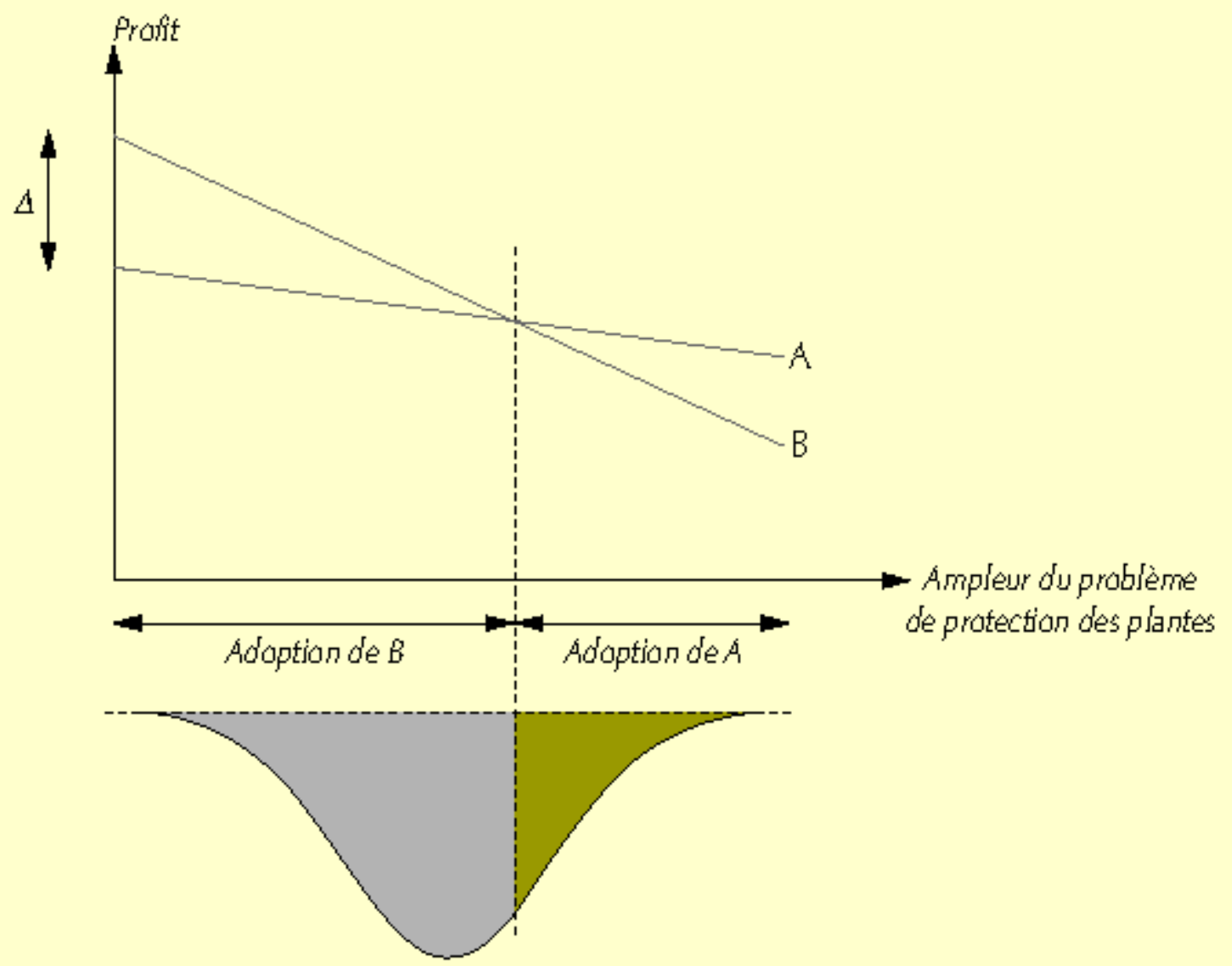

$\Delta$ : coj̀t supplementaire de la pratique $A$ par rapport à la pratique $B$

Figure 1. Représentation graphique de la comparaison entre différents programmes de protection des plantes. 


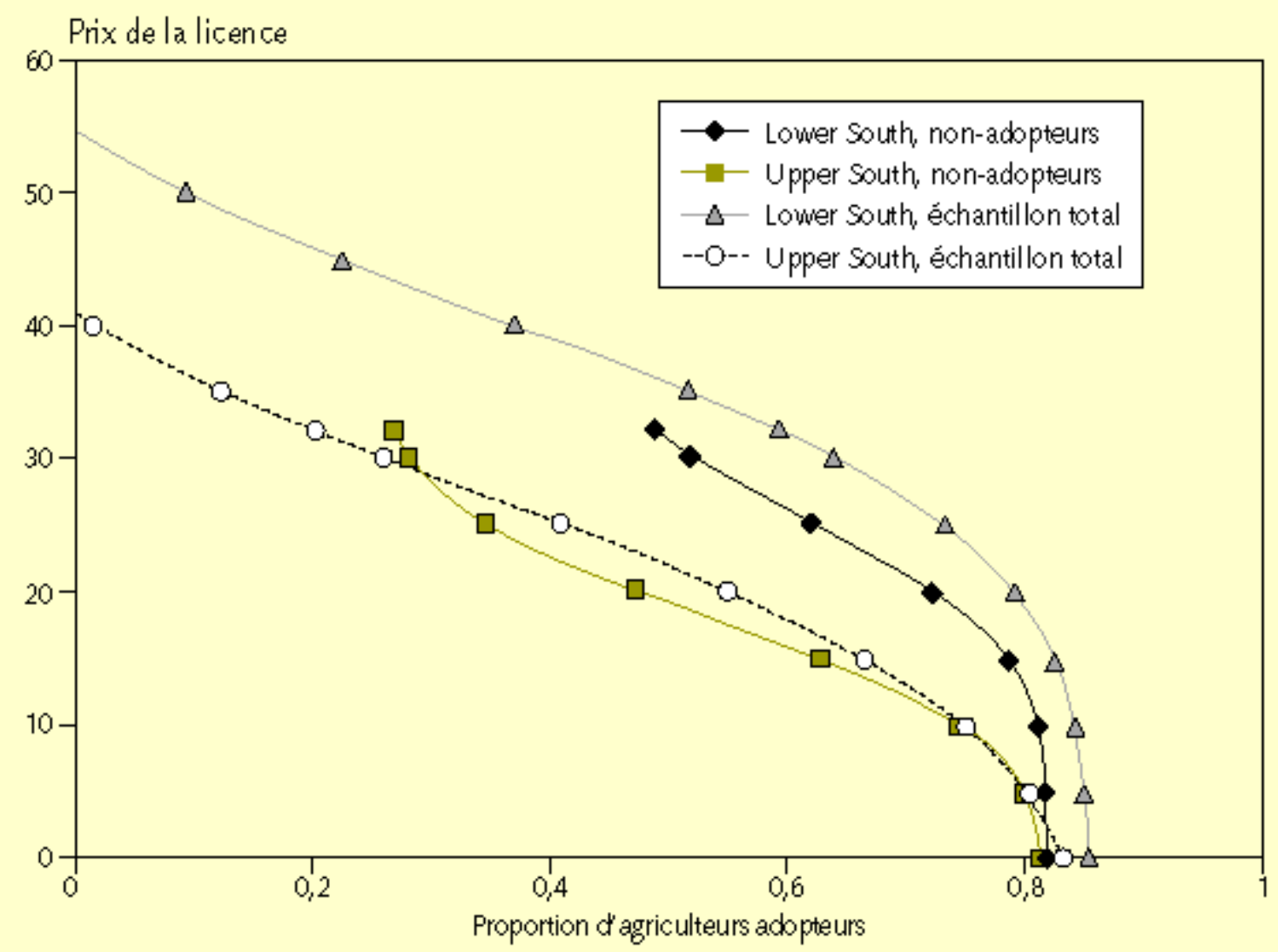

Figure 2. Supplément de prix sur la semence OGM et diffusion du coton IR (d'après Hubbell et al. [6]).

Tableau 1. Remplacement des programmes de protection des plantes.

\begin{tabular}{|c|c|c}
\hline $\begin{array}{c}\text { Type de progra mme } \\
\text { de protection des plantes }\end{array}$ & Cas HT & Cas IR \\
\hline & Semence conventionnelle & Semence conventionnelle \\
Conventionnel intensif & + & + \\
& Herbicide sélectif & Insecticides \\
OGM & Semence HT & Semence IR \\
& + & + \\
& Herbicide total & Insecticide additionne** \\
\hline
\end{tabular}

* Dans certains cas (ex. : cotton) la semence OGM n'offre pas une résistance totale face à tou s les insectes, et certains insec ticides additionnels peuvent être nécessaires si les insectes non ciblés par la semence OGM posent des problèmes assez importants. 
Tableau 2. Suppléments de prix des semences OGM par rapport aux semences conventionnelles.

\begin{tabular}{|c|c|c|}
\hline \multicolumn{2}{|c|}{ Supplément de prix sur la semence } & \multirow[t]{2}{*}{ Principaux événe ments concernés } \\
\hline$\$ /$ acre & FF/ha & \\
\hline$>30$ & $>550$ & $\mathbb{R}$ (coton) \\
\hline \multirow[t]{2}{*}{$7-11$} & $125-200$ & IR (maïs) \\
\hline & & HT-RR* (soja, maïs, colza) \\
\hline 0 & 0 & HT-LL* (colza et maīs) \\
\hline
\end{tabular}

* RR désigne la tolérance au Roundup (glyphosate) et LL désigne la tolérance au Liberty (glu fosinate).

Tableau 3. Diffusion des OGM aux Etats-Unis (\%).

\begin{tabular}{|lcccccc}
\hline & & $\mathbf{1 9 9 6}$ & $\mathbf{1 9 9 7}$ & $\mathbf{1 9 9 8}$ & $\mathbf{1 9 9 9}$ & $\mathbf{2 0 0 0}$ \\
\hline Soja & HT & 7 & 17 & 44 & 57 & 52 \\
Mais & HT & & 1 & 5 & 4 & 4 \\
& IR & 1 & 8 & 20 & 25 & 19 \\
& HT + IR & & - & 4 & 4 & 2 \\
Coton & HT & & 10 & 15 & 28 & 20 \\
& IR & 15 & 15 & 15 & 16 & 17 \\
& HT + IR & & 1 & 10 & 11 & 11
\end{tabular}

Tableau 4. Impact de l'adoption des OGM mesuré à partir de l'enquête ARMS.

\begin{tabular}{|lccc}
\hline & $\begin{array}{c}\text { Soja HT } \\
(\mathbf{1 9 9 7 )}\end{array}$ & $\begin{array}{c}\text { Coton HT } \\
(\mathbf{1 9 9 7})\end{array}$ & $\begin{array}{c}\text { Coton IR } \\
(\mathbf{1 9 9 7})\end{array}$ \\
\hline Rendement & $(+)$ & + & + \\
Marge nette & 0 & + & + \\
Herbicide sélectif & - & 0 & \\
Glyphosate & + & 0 & \\
Insecticides & & & - \\
\hline
\end{tabular}

D'après Femandez-Cornejo et McBride [4].

0 : effet non sign ificatif; $(+)$ : acc roissement faible mais significatif; + : acc roissement significatif; - diminution significative. Les don nées sur le coton IR ne concement que les États du Sud-Est.

* La marge nette prend en compte l'économie sur k temps de travail. 